\title{
Effective Hamiltonian for striped and paired states at the half-filled Landau level
}

\author{
Nobuki Maeda \\ Department of Physics, Hokkaido University, Sapporo 060-0810, Japan
}

\begin{abstract}
We study a pairing mechanism for the quantum Hall system using a mean field theory with a basis on the von Neumann lattice, on which the magnetic translations commute. In the Hartree-FockBogoliubov approximation, we solve the gap equation for spin-polarized electrons at the half-filled Landau levels. We obtain an effective Hamiltonian which shows a continuous transition from the compressible striped state to the paired state. Furthermore, a crossover occurs in the pairing phase. The energy spectrum and energy gap of the quasiparticle in the paired state is calculated numerically at the half-filled second Landau level.
\end{abstract}

PACS numbers: 73.40.Hm; 74.20.Fg; 71.45.Lr

\section{INTRODUCTION}

The fractional quantum Hall effect (FQHE) [1] is observed around rational filling factors with odd denominators in the lowest Landau level. This effect is caused by the formation of the incompressible liquid state [2]. Recent experiments showed that the compressible state around the half-filled lowest Landau level is similar to the Fermi liquid. In the composite fermion theory [3], this Fermi-liquid-like state is described by the composite of an electron and two flux quanta, which behaves like a free electron with no magnetic field in the mean field approximation.

In the second Landau level, the situation dramatically changes; the FQHE is observed around the half-filling [4]. The origin of this quantum Hall state is still mysterious. Recent numerical works [5.6] seem to support the Pfaffian state [7] 9], which has the spin-polarized p-wave pairing potential, rather than the spin-singlet paired state 10. However the microscopic explanation for this pairing mechanism is not known yet. More quantitative search is, therefore, needed on the basis of the microscopic BCS-like model. In the present paper, we study the paired state in the mean field theory starting from a microscopic Hamiltonian.

It was shown that the Fermi-liquid-like state [11] can be constructed at an arbitrary filling factor in the von Neumann lattice formalism [12. In a strong magnetic field, the free kinetic energy is quenched and the kinetic energy is generated from the Coulomb interaction. If the translational symmetry on the von Neumann lattice and U(1) symmetry are unbroken, the Fermi surface is formed in the magnetic Brillouin zone in the mean field theory. In the Hartree-Fock approximation, this state corresponds to the striped state [13] which is observed at half-filled second and higher Landau levels [14,15]. In Ref. [6], the first order transition from striped state to paired state is obtained in the numerical calculation of small systems. We regard this striped state as the normal state and examine the U(1) symmetry breaking mechanism. The hopping potential $\varepsilon(p)$ and gap potential $\Delta(p)$ are determined self-consistently in the Hartree-Fock-Bogoliubov approximation.

Naively it seems that the formation of the paired state by only the repulsive force is impossible in the mean field theory. However, screening effect could make the potential attractive for the pairing. In fact, the quantum effect of fermion loop screens the Coulomb potential and the pseudopotential in the Landau level space becomes attractive. Depending on strength of the screening effect, there are two phases, that is, stripe phase and pairing phase. On the basis of this observation, we construct an effective Hamiltonian which shows a transition from the striped state to the paired state. The transition is continuous and very smooth. The energy spectrum of the quasiparticle is obtained and energy gap is calculated numerically. Furthermore, we find a crossover from the pairing phase to the gap-dominant pairing phase in which the low-energy excitation occurs around zeros of the gap potential.

The paper is organized as follows. In Sec. II, a mean field theory on the von Neumann lattice is presented and self-consistency equations are obtained. In Sec. III, we analyze the self-consistency equations and a screening effect. It is shown that there are a stripe phase and pairing phase depending on strength of the screening effect. In Sec. IV, we present an effective Hamiltonian which shows a transition from the striped state to the paired state and calculate the energy spectrum of the quasiparticle. Summary and discussion are given in Sec. V. 


\section{HARTREE-FOCK-BOGOLIUBOV APPROXIMATION}

We consider two-dimensional electron systems in the presence of a perpendicular uniform magnetic field $B$. The free particle energy is quenched to the Landau level energy $E_{l}=\hbar \omega_{c}(l+1 / 2), l=0,1,2 \ldots$, where $\omega_{c}=e B / m_{e}($ the cyclotron frequency). We suppose that the electrons are spin-polarized and ignore the spin degree of freedom. In this system, the translation is generated by the magnetic translation operators $T(x, y)$ with displacement vector $(x, y)$. $T(x, y)$ commutes with the Hamiltonian. However, magnetic translation operators are non-commutative, that is,

$$
T\left(x_{1}, y_{1}\right) T\left(x_{2}, y_{2}\right)=e^{i \frac{e B}{\hbar}\left(x_{1} y_{2}-x_{2} y_{1}\right)} T\left(x_{2}, y_{2}\right) T\left(x_{1}, y_{1}\right)
$$

Therefore, the translation becomes commutative if we restrict the displacement vectors on a two-dimensional lattice which has a unit cell with an area $2 \pi \hbar / e B$. This lattice is called the von Neumann lattice. We consider lattice sites $a(m, n)$, where $m, n$ are integers and lattice spacing $a=\sqrt{2 \pi \hbar / e B}$. Then $T(m a, n a)$ 's commute with each other and have simultaneous eigenstates as

$$
T(m a, n a) u_{l, p}(\mathbf{r})=e^{i\left(p_{x} m+p_{y} n\right) a / \hbar} u_{l, p}(\mathbf{r})
$$

in the $l$ th Landau level. We call $\mathbf{p}=\left(p_{x}, p_{y}\right)$ the momentum and $u_{l, p}(\mathbf{r})$ the Bloch wave on the von Neumann lattice, which is defined on the Brillouin zone $\left|p_{x}\right|,\left|p_{y}\right|<\pi \hbar / a$. It was proved that the eigenstates form an orthogonal complete set [16,12]. For simplicity, we set $a=\hbar=c=1$.

The Bloch wave $u_{l, p}(\mathbf{r})$ is constructed by using the coherent states of the guiding center coordinates $(X, Y)$ defined by

$$
\begin{aligned}
X & =x-\xi \\
Y & =y-\eta \\
\xi & =\frac{1}{e B}\left(-i \frac{\partial}{\partial y}+e A_{y}\right), \\
\eta & =-\frac{1}{e B}\left(-i \frac{\partial}{\partial x}+e A_{x}\right),
\end{aligned}
$$

where $\nabla \times A=B$ and $(\xi, \eta)$ are called the relative coordinates. These operators satisfy the following commutation relations,

$$
\begin{gathered}
{[\xi, \eta]=-[X, Y]=-\frac{i}{e B},} \\
{[\xi, X]=[\xi, Y]=[\eta, X]=[\eta, Y]=0}
\end{gathered}
$$

The coherent states of $(X, Y)$ are defined by

$$
\begin{gathered}
(X+i Y) f_{l m n}(\mathbf{r})=(m+i n) f_{l m n}(\mathbf{r}) \\
\frac{m_{e} \omega_{c}^{2}}{2}\left(\xi^{2}+\eta^{2}\right) f_{l m n}(\mathbf{r})=E_{l} f_{l m n}(\mathbf{r}) .
\end{gathered}
$$

Note that these coherent states are non-orthogonal complete set as

$$
\int d^{2} r f_{l, m^{\prime}+m, n^{\prime}+n}(\mathbf{r}) f_{l^{\prime}, m^{\prime}, n^{\prime}}(\mathbf{r})=\delta_{l l^{\prime}} e^{i \pi(m+n+m n)-\frac{\pi}{2}\left(m^{2}+n^{2}\right)} .
$$

Using $f_{l m n}(\mathbf{r})$, the orthogonal basis $u_{l, p}(\mathbf{r})$ is given by

$$
u_{l, p}(\mathbf{r})=\frac{1}{\beta(p)} \sum_{m n} e^{i p_{x} m+i p_{y} n} f_{l m n}(\mathbf{r})
$$

where $\beta(p)$ is a normalization factor [12]. In this basis, two-dimensional momentum is a good quantum number and the Fermi surface is formed in the mean field theory [11]. It was shown that the mean field state could explain the anisotropic compressible state observed at the half-filled second and higher Landau levels [13. Therefore the basis on the von Neumann lattice is useful tool to investigate the physics at the half-filled second and higher Landau levels.

Fourier transforming the Bloch wave, we can obtain the orthogonal localized basis [17] 


$$
w_{l, \mathbf{X}}(\mathbf{r})=\int_{\mathrm{BZ}} \frac{d^{2} p}{(2 \pi)^{2}} u_{l, p}(\mathbf{r}) e^{-i\left(p_{x} m+p_{y} n\right)+i \lambda(p)}
$$

where $\mathbf{X}=(m, n)$, BZ stands for the Brillouin zone, and $\lambda(p)$ represents the gauge degree of freedom which is taken to satisfy the periodic condition in the Brillouin zone. We call this basis the Wannier basis. This basis is localized on a position of the lattice site. The explicit forms of these bases are given in Ref. [12].

Using the Bloch wave basis and Wannier basis, we can expand the electron field operator $\psi(\mathbf{r})$ as

$$
\begin{aligned}
\psi(\mathbf{r}) & =\sum_{l=0}^{\infty} \int_{\mathrm{BZ}} \frac{d^{2} p}{(2 \pi)^{2}} a_{l}(p) u_{l, p}(\mathbf{r}) \\
& =\sum_{l=0}^{\infty} \sum_{\mathbf{X}} b_{l}(\mathbf{X}) w_{l, \mathbf{X}}(\mathbf{r})
\end{aligned}
$$

where $a_{l}(p)$ and $b_{l}(\mathbf{X})$ are anti-commuting annihilation operators in the momentum space and lattice space, respectively. The total Hamiltonian of the present system is

$$
H=\int d^{2} r \psi^{\dagger}(\mathbf{r}) \frac{(-i \nabla+e A)^{2}}{2 m_{e}} \psi(\mathbf{r})+\frac{1}{2} \int d^{2} r d^{2} r^{\prime}:\left(\rho(\mathbf{r})-\rho_{0}\right) V\left(\mathbf{r}-\mathbf{r}^{\prime}\right)\left(\rho\left(\mathbf{r}^{\prime}\right)-\rho_{0}\right):,
$$

where colons mean the normal ordering, $\rho(\mathbf{r})=\psi^{\dagger}(\mathbf{r}) \psi(\mathbf{r}), V(\mathbf{r})=q^{2} / r$, and $\rho_{0}$ is the uniform background density.

Let us project the system to the $l$ th Landau level space. Up to the Landau level energy $E_{l}$, the projected Hamiltonian $H^{(l)}$ is written as

$$
H^{(l)}=\frac{1}{2} \sum_{X_{1} X_{1}^{\prime} X_{2} X_{2}^{\prime}}: b_{l}^{\dagger}\left(\mathbf{X}_{1}\right) b_{l}\left(\mathbf{X}_{1}^{\prime}\right) V_{l}(\mathbf{X}, \mathbf{Y}, \mathbf{Z}) b_{l}^{\dagger}\left(\mathbf{X}_{2}\right) b_{l}\left(\mathbf{X}_{2}^{\prime}\right):
$$

where $\mathbf{X}=\mathbf{X}_{1}-\mathbf{X}_{1}^{\prime}, \mathbf{Y}=\mathbf{X}_{2}-\mathbf{X}_{2}^{\prime}, \mathbf{Z}=\mathbf{X}_{1}-\mathbf{X}_{2}^{\prime}$, and $V_{l}(\mathbf{X}, \mathbf{Y}, \mathbf{Z})$ is given as

$$
\begin{aligned}
V_{l}(\mathbf{X}, \mathbf{Y}, \mathbf{Z}) & =\int \frac{d^{2} k}{(2 \pi)^{2}} \int_{\mathrm{BZ}} \frac{d^{2} p_{1}}{(2 \pi)^{2}} \frac{d^{2} p_{2}}{(2 \pi)^{2}} \tilde{v}_{l}(k) e^{i\left[p_{1} \cdot X+p_{2} \cdot Y+k \cdot Z+f\left(p_{1}, p_{1}+k\right)+f\left(p_{2}+k, p_{2}\right)\right]}, \\
f(p+k, p) & =\int_{p}^{p+k}\left(\alpha\left(p^{\prime}\right)+\nabla_{p^{\prime}} \lambda\left(p^{\prime}\right)\right) d p^{\prime} .
\end{aligned}
$$

Here $\alpha(p)$ is a vector potential, $\nabla_{p}=\left(\frac{\partial}{\partial p_{x}}, \frac{\partial}{\partial p_{y}}\right), \nabla_{p} \times \alpha(p)=-1 / 2 \pi$ which is a unit flux penetrating the momentum space, and the line integral is along a straight line. The effective Coulomb potential in the $l$ th Landau level $\tilde{v}_{l}$ is given by

$$
\tilde{v}_{l}(k)=\left\{L_{l}\left(\frac{k^{2}}{4 \pi}\right)\right\}^{2} e^{-\frac{k^{2}}{4 \pi}} \frac{2 \pi q^{2}}{k},
$$

and $\tilde{v}_{l}(0)=0$ due to the charge neutrality condition, where $L_{l}$ is the Laguerre polynomial.

The system is translationally invariant in the lattice space and in the momentum space with the uniform magnetic field $\nabla \times \alpha(p)$. The translational symmetry in the momentum space is called the K-invariance. We consider the case that the K-invariance is spontaneously broken and the kinetic term is induced through the correlation effect.

We apply the mean field approximation to the projected Hamiltonian (2.12). Let us consider the following mean fields, which are translationally invariant on the von Neumann lattice,

$$
\begin{aligned}
U_{l}\left(\mathbf{X}-\mathbf{X}^{\prime}\right) & =\left\langle b_{l}^{\dagger}\left(\mathbf{X}^{\prime}\right) b_{l}(\mathbf{X})\right\rangle, \\
U_{l}^{(+)}\left(\mathbf{X}-\mathbf{X}^{\prime}\right) & =\left\langle b_{l}^{\dagger}\left(\mathbf{X}^{\prime}\right) b_{l}^{\dagger}(\mathbf{X})\right\rangle, \\
U_{l}^{(-)}\left(\mathbf{X}-\mathbf{X}^{\prime}\right) & =\left\langle b_{l}\left(\mathbf{X}^{\prime}\right) b_{l}(\mathbf{X})\right\rangle .
\end{aligned}
$$

These mean fields break the K-invariance and U(1) symmetry. $U_{l}^{( \pm)}$satisfies $U_{l}^{(-) *}(\mathbf{X})=U_{l}^{(+)}(-\mathbf{X})$ and $U_{l}^{(-)}(-\mathbf{X})=$ $-U_{l}^{(-)}(\mathbf{X})$. Using these mean fields, we approximate $H^{(l)}$ as 


$$
\begin{aligned}
& H_{\text {mean }}^{(l)}= \sum_{X X^{\prime}} U_{l}\left(\mathbf{X}-\mathbf{X}^{\prime}\right)\left\{v_{l}^{H}\left(\mathbf{X}^{\prime}-\mathbf{X}^{\prime}\right)-v_{l}^{F}\left(\mathbf{X}^{\prime}-\mathbf{X}\right)\right\} b^{\dagger}(\mathbf{X}) b\left(\mathbf{X}^{\prime}\right) \\
&+\frac{1}{2} \sum_{X X^{\prime} Y}\left\{U_{l}^{(+)}(\mathbf{Y}) V_{l}^{B}\left(\mathbf{X}-\mathbf{X}^{\prime}, \mathbf{Y}\right) b_{l}(\mathbf{X}) b_{l}\left(\mathbf{X}^{\prime}\right)\right. \\
&\left.+U_{l}^{(-)}(\mathbf{Y}) V_{l}^{B}\left(-\mathbf{Y}, \mathbf{X}^{\prime}-\mathbf{X}\right) b_{l}^{\dagger}(\mathbf{X}) b_{l}^{\dagger}\left(\mathbf{X}^{\prime}\right)\right\} .
\end{aligned}
$$

Here the Hartree potential $v_{l}^{H}$ and Fock potential $v_{l}^{F}$ are given by the following sum rules

$$
\begin{aligned}
\sum_{Z} V_{l}(\mathbf{X}, \mathbf{Y}, \mathbf{Z}) & =v_{l}^{H}(\mathbf{X}) \delta_{-\mathbf{Y}}^{\mathbf{X}}, \\
\sum_{X} V_{l}(\mathbf{X}, \mathbf{Y}-\mathbf{X}, \mathbf{Z}) & =v_{l}^{F}(\mathbf{Z}) \delta_{0}^{\mathbf{Y}}
\end{aligned}
$$

where

$$
\begin{aligned}
v_{l}^{H}(\mathbf{X}) & =\tilde{v}_{l}(2 \pi \mathbf{X}), \\
v_{l}^{F}(\mathbf{X}) & =\int \frac{d^{2} k}{(2 \pi)^{2}} \tilde{v}_{l}(k) e^{i k \cdot X} .
\end{aligned}
$$

We define the Hartree-Fock potential as $v_{l}^{\mathrm{HF}}(\mathbf{X})=v_{l}^{H}(\mathbf{X})-v_{l}^{F}(\mathbf{X})$ and its Fourier transform as $\tilde{v}_{l}^{\mathrm{HF}}(p)$. The Bogoliubov potential $V_{l}^{B}$ is written as

$$
\begin{aligned}
V_{l}^{B}(\mathbf{X}, \mathbf{Y}) & =\sum_{Z} V_{l}(\mathbf{X}+\mathbf{Z}, \mathbf{Y}+\mathbf{Z}, \mathbf{Z}) \\
& =\int_{\mathrm{BZ}} \frac{d^{2} p_{1}}{(2 \pi)^{2}} \frac{d^{2} p_{2}}{(2 \pi)^{2}} \tilde{V}_{l}^{B}\left(p_{1}, p_{2}\right) e^{i p_{1} \cdot X+i p_{2} \cdot Y}
\end{aligned}
$$

and $\tilde{V}_{l}^{B}\left(p_{1}, p_{2}\right)$ is given by

$$
\tilde{V}_{l}^{B}\left(p_{1}, p_{2}\right)=\sum_{N} \tilde{v}_{l}\left(p_{1}+p_{2}+2 \pi N\right) e^{i\left[f\left(p_{1},-p_{2}-2 \pi N\right)+f\left(-p_{1}, p_{2}+2 \pi N\right)\right]}
$$

where $N=\left(N_{x}, N_{y}\right), N_{x}, N_{y}$ are integers. Then we introduce the hopping potential $\varepsilon$ and gap potential $\Delta$ as

$$
\begin{aligned}
\varepsilon_{l, \mathbf{X}} & =U_{l}(\mathbf{X}) v_{l}^{H F}(-\mathbf{X}), \\
\Delta_{l, \mathbf{X}} & =\sum_{Y} U_{l}^{(-)}(\mathbf{Y}) V_{l}^{B}(-\mathbf{X},-\mathbf{Y}) .
\end{aligned}
$$

We define these Fourier transforms as $\varepsilon_{l}(p)$ and $\Delta_{l}(p) e^{-i(\lambda(p)+\lambda(-p))}$, respectively. The phase factor of the gap potential is the same in Eq. (2.9). $V_{l}^{B}$ satisfies $V_{l}^{B}(-\mathbf{X},-\mathbf{Y})=V_{l}^{B}(\mathbf{X}, \mathbf{Y})$ and we have $\Delta_{l,-X}=-\Delta_{l, X}$. Using these potentials, the mean field Hamiltonian reads

$$
H_{\text {mean }}^{(l)}=\sum_{X X^{\prime}}\left[\varepsilon_{l, \mathbf{X}-\mathbf{X}^{\prime}} b_{l}^{\dagger}(\mathbf{X}) b_{l}\left(\mathbf{X}^{\prime}\right)+\frac{1}{2} \Delta_{l, \mathbf{X}^{\prime}-\mathbf{X}}^{*} b(\mathbf{X}) b\left(\mathbf{X}^{\prime}\right)+\frac{1}{2} \Delta_{l, \mathbf{X}^{\prime}-\mathbf{X}} b^{\dagger}\left(\mathbf{X}^{\prime}\right) b^{\dagger}(\mathbf{X})\right] .
$$

This Hamiltonian is diagonalized by the Bogoliubov transformation in the momentum space. We fix the gauge of vector potential $\alpha$ in the momentum space as $\alpha(p)=\left(p_{y} / 2 \pi, 0\right)$. In this gauge, the boundary conditions in the Brillouin zone are given by

$$
\begin{aligned}
a_{l}(p+2 \pi N) & =e^{i \pi\left(N_{x}+N_{y}\right)-i N_{y} p_{x}} a_{l}(p), \\
\Delta_{l}(p+2 \pi N) & =e^{-2 i N_{y} p_{x}} \Delta_{l}(p) .
\end{aligned}
$$

Equation (2.28) and the relation $\Delta_{l}(-p)=-\Delta_{l}(p)$ make the gap potential $\Delta_{l}(p)$ have four zeros at $\mathbf{p}=(0,0),(0, \pi)$, $(\pi, 0)$, and $(\pi, \pi)$. We assume $\varepsilon_{l}(-p)=\varepsilon_{l}(p) . U_{l}$ and $U_{l}^{(-)}$are calculated by using $H_{\text {mean }}^{(l)}$. Then, the hopping potential and gap potential in Eqs. (2.24) and (2.25) are determined by the self-consistency equations, 


$$
\begin{aligned}
\xi_{l}(p) & =\int_{\mathbf{B Z}} \frac{d^{2} p_{1}}{(2 \pi)^{2}} \frac{E_{l}\left(p_{1}\right)-\xi_{l}\left(p_{1}\right)}{2 E_{l}\left(p_{1}\right)} \tilde{v}_{l}^{\mathrm{HF}}\left(p_{1}-p\right)-\mu, \\
\Delta_{l}(p) & =-\int \frac{d^{2} p_{1}}{(2 \pi)^{2}} \frac{\Delta_{l}\left(p_{1}\right)}{2 E_{l}\left(p_{1}\right)} \tilde{v}_{l}\left(p_{1}-p\right) e^{i \int_{p}^{p_{1}} 2 \alpha(k) d k},
\end{aligned}
$$

where $\mu$ is the chemical potential and $E_{l}(p)$ is the spectrum of the quasiparticle which is defined by

$$
\begin{aligned}
E_{l}(p) & =\sqrt{\xi_{l}(p)^{2}+\left|\Delta_{l}(p)\right|^{2}}, \\
\xi_{l}(p) & =\varepsilon_{l}(p)-\mu .
\end{aligned}
$$

These self-consistency equations are equivalent to sum up infinite diagrams of the Fermion self-energy part. Note that the integral region of Eq. (2.30) are infinite. Note also that the gauge field appears in the gap equation (2.30). The gauge field represents two unit flux on the Brillouin zone. This corresponds to the flux carried by the Cooper pair $\langle a(p) a(-p)\rangle$ which is separated between $p$ and $-p$ in the Brillouin zone. The mean field $\left\langle a^{\dagger}(p) a(p)\right\rangle$, on the other hand, is placed on the same point. Therefore no gauge field appears in Eq. (2.29).

The filling factor of the $l$ th Landau level is denoted by $\nu_{l}$ and total filling factor is given by $\nu=l+\nu_{l}$. Using the mean fields, the filling factor is given by

$$
\nu_{l}=\frac{1}{2}-\int_{\mathbf{B Z}} \frac{d^{2} p}{(2 \pi)^{2}} \frac{\xi_{l}(p)}{2 E_{l}(p)} .
$$

In the limit of $\Delta \rightarrow 0, \nu_{l}$ is equal to $\int_{\mathbf{B Z}} \theta\left(\mu-\varepsilon_{l}(p)\right) d^{2} p /(2 \pi)^{2}$.

\section{STRIPE PHASE AND PAIRING PHASE}

In this section, we analyze the self-consistency equations (2.29) and (2.30). It is shown that the screening effect plays important roles for the pairing mechanism. For simplicity, we omit the Landau level index $l$ and use $q^{2} / a$ as the unit of energy.

It is convenient for solving Eq. (2.29) to use the following mode expansions,

$$
\begin{aligned}
\xi(p) & =\sum_{X} \xi_{\mathbf{X}} e^{i X \cdot p}, \\
\frac{\xi(p)}{E(p)} & =\sum_{X} \eta_{\mathbf{X}} e^{i X \cdot p},
\end{aligned}
$$

where $\xi_{-\mathbf{X}}=\xi_{\mathbf{X}}, \eta_{-\mathbf{X}}=\eta_{\mathbf{X}}$, and $\xi_{\mathbf{X}}, \eta_{\mathbf{X}}$ are real numbers. Then Eq. 2.29) becomes

$$
\begin{aligned}
& \xi_{\mathbf{X}}=-\frac{v^{\mathrm{HF}}(\mathbf{X})}{2} \eta_{\mathbf{X}}, \text { for } \mathbf{X} \neq 0, \\
& \xi_{\mathbf{0}}=-\frac{v^{\mathrm{HF}}(\mathbf{0})}{2}\left(\eta_{\mathbf{0}}-1\right)-\mu .
\end{aligned}
$$

The zero mode $\eta_{\mathbf{0}}$ is related to the filling factor as $\nu_{l}=\left(1-\eta_{\mathbf{0}}\right) / 2$ by Eq. (2.33). Therefore the zero mode $\xi_{0}$ is determined by $\mu$ and $\nu_{l}$. In particular, $\eta_{\mathbf{0}}=0$ for the half-filling case $\left(\nu_{l}=1 / 2\right)$. We introduce hopping parameters $t_{\mathbf{X}}=-2 \xi_{\mathbf{X}}$ and regard $\xi_{\mathbf{X}}$ as a functional of $t_{\mathbf{X}}$ 's and $\Delta(p)$. Then the self-consistency for $t_{\mathbf{X}}$ is written as

$$
t_{\mathbf{X}}=-2 \xi \mathbf{X}\left[\left\{t_{(m, n)}\right\}, \Delta(p)\right] .
$$

Using $t_{(m, n)}$, the hopping term is written by $-\sum_{m, n \geq 0} t_{(m, n)} \cos \left(p_{x} m+p_{y} n\right) a^{\dagger}(p) a(p)$.

Next we discuss the self-consistency for the gap potential $\Delta(p)$. Eq. (2.30) is rewritten as

$$
\Delta(p)=-\int \frac{d^{2} k}{(2 \pi)^{2}} \tilde{v}(k) e^{i k \cdot D} \frac{\Delta(p)}{2 E(p)},
$$

where $\mathbf{D}=\left(-i \frac{\partial}{\partial p_{x}}+2 \alpha_{x},-i \frac{\partial}{\partial p_{y}}+2 \alpha_{y}\right)$. It is convenient to introduce eigenfunctions of the operator $\mathbf{D}^{2}$, that is, $\mathbf{D}^{2} \psi_{n}(p)=e_{n} \psi_{n}(p)$ with $e_{n}=(2 n+1) / \pi, n=0,1,2, \ldots$ The index $n$ labels the $n$th Landau level in the momentum 
space. The eigenfunctions which obey the same boundary condition as the gap function are doubly degenerate and are given by

$$
\begin{aligned}
\psi_{n}^{(2)}(p) & =N_{n} \sum_{N_{y}} H_{n}\left(\frac{p_{y}+\left(2 N_{y}+1\right) \pi}{\sqrt{2 \pi}}\right) e^{i\left(2 N_{y}+1\right) p_{x}-\frac{1}{2 \pi}\left(p_{y}+\left(2 N_{y}+1\right) \pi\right)^{2}} \\
& =\frac{2 \pi}{\sqrt{n !}}\left(\frac{\pi}{2}\right)^{n / 2}\left(D_{x}-i D_{y}\right)^{n} \psi_{0}^{(2)}(p), \\
\psi_{n}^{(3)}(p) & =N_{n} \sum_{N_{y}} H_{n}\left(\frac{p_{y}+2 N_{y} \pi}{\sqrt{2 \pi}}\right) e^{i 2 N_{y} p_{x}-\frac{1}{2 \pi}\left(p_{y}+2 N_{y} \pi\right)^{2}} \\
& =\frac{2 \pi}{\sqrt{n !}}\left(\frac{\pi}{2}\right)^{n / 2}\left(D_{x}-i D_{y}\right)^{n} \psi_{0}^{(3)}(p),
\end{aligned}
$$

where $H_{n}$ is the Hermite polynomial, $N_{n}=1 / \sqrt{2^{n-1} n !}$, and $\psi_{0}^{(2)}, \psi_{0}^{(3)}$ are written by theta functions as

$$
\begin{aligned}
& \psi_{0}^{(2)}(p)=N_{0} e^{-\frac{p_{y}^{2}}{2 \pi}} \vartheta_{2}\left(\frac{p_{x}+i p_{y}}{\pi} \mid 2 i\right), \\
& \psi_{0}^{(3)}(p)=N_{0} e^{-\frac{p_{y}^{2}}{2 \pi}} \vartheta_{3}\left(\frac{p_{x}+i p_{y}}{\pi} \mid 2 i\right) .
\end{aligned}
$$

These eigenfunctions have the parity symmetry, $\psi_{n}(-p)=(-)^{n} \psi_{n}(p)$. Therefore the gap potential can be expanded by $\psi_{2 n+1}(p)$. Furthermore the eigenfunctions satisfy the following relations

$$
\begin{aligned}
& \psi_{n}^{(2)}\left(p_{x}+\pi, p_{y}\right)=-\psi_{n}^{(2)}\left(p_{x}, p_{y}\right), \\
& \psi_{n}^{(3)}\left(p_{x}+\pi, p_{y}\right)=\psi_{n}^{(3)}\left(p_{x}, p_{y}\right), \\
& \psi_{n}^{(2)}\left(p_{x}, p_{y}+\pi\right)=e^{-i p_{x}} \psi_{n}^{(3)}\left(p_{x}, p_{y}\right), \\
& \psi_{n}^{(3)}\left(p_{x}, p_{y}+\pi\right)=e^{-i p_{x}} \psi_{n}^{(2)}\left(p_{x}, p_{y}\right),
\end{aligned}
$$

and $\psi_{n}(\pi m, \pi n)=0$. Moreover $\psi_{n}^{(2)}(\pi n / 2, \pi)=0$ and $\psi_{n}^{(3)}(\pi n / 2,0)=0$. We can expand the gap potential as

$$
\begin{aligned}
& \Delta(p)=\sum_{n \geq 1, i=2,3} c_{n}^{(i)} \psi_{2 n-1}^{(i)}(p), \\
& \frac{\Delta(p)}{E(p)}=\sum_{n \geq 1, i=2,3} d_{n}^{(i)} \psi_{2 n-1}^{(i)}(p) .
\end{aligned}
$$

Then Eq. (2.30) becomes

$$
\begin{aligned}
c_{n}^{(i)} & =-\frac{F_{2 n+1}}{2} d_{n}^{(i)}, \\
F_{n} & =\int \frac{d^{2} k}{(2 \pi)^{2}} \tilde{v}(k) L_{n}\left(\frac{k^{2}}{2 \pi}\right) e^{-\frac{k^{2}}{4 \pi}} .
\end{aligned}
$$

Note that although $F_{n}$ 's have the same form as the Haldane's pseudopotentials [20], the physical meaning is different. Haldane's one is the 2-body interaction projected to the relative angular momentum in the single Landau level. It is not screened by definition. The potential $F_{n}$, on the other hand, is a potential appearing in the gap equation which is renormalized by higher order corrections. Actually the potential $F_{n}$ is screened by the polarization $\Pi(p)$ due to the Fermion loop diagrams. We approximate the screened potential as

$$
\begin{aligned}
\tilde{v}\left(p, m_{\mathrm{TF}}\right) & =1 /\left(\tilde{v}(p)^{-1}+m_{\mathrm{TF}}\right), \\
m_{\mathrm{TF}} & =-\Pi(0),
\end{aligned}
$$

where $m_{\mathrm{TF}}$ is the Thomas-Fermi mass. Calculating the one-loop diagram, $m_{\mathrm{TF}}$ is given by

$$
m_{\mathrm{TF}}=\int_{\mathbf{B Z}} \frac{d^{2} p}{(2 \pi)^{2}} \frac{|\Delta(p)|^{2}}{2 E(p)^{3}}
$$


We use the screened potential $\tilde{v}\left(p, m_{\mathrm{TF}}\right)$ in the Fock potential (2.21) and Bogoliubov potential (2.23). In the zero limit of the gap potential $\Delta, m_{\mathrm{TF}}$ becomes the density of states on the Fermi surface. It should be noted that the Hartree potential $v^{H}$ is not screened because the polarization effect is automatically included in the self-consistency equation.

The $m_{\mathrm{TF}}$ dependences of the hopping strength $v^{\mathrm{HF}}(\mathbf{X})$ and pseudopotentials $F_{n}$ are plotted in Figs. (1)-(6) for $l=0,1,2$. As seen in these figures, potentials change their signs at $m_{\mathrm{TF}} \approx 1.0 \sim 2.0$. Especially for $l=1$ and 2 , all potentials change their signs together at large $m_{\mathrm{TF}}$. This observation leads us to a strong statement for $l=1$ and 2 . Using Eqs. (3.1)-(3.4) and Eqs. (3.15)- (3.18), the following inequalities are concluded at $\nu_{l}=1 / 2$,

$$
\begin{gathered}
\int \frac{\xi(p)^{2}}{E(p)} \frac{d^{2} k}{(2 \pi)^{2}}=-\sum_{X \neq 0} \frac{v^{\mathrm{HF}}(\mathbf{X})}{2} \eta_{\mathbf{X}}^{2} \geq 0, \\
\int \frac{|\Delta(p)|^{2}}{E(p)} \frac{d^{2} k}{(2 \pi)^{2}}=-\sum_{n \geq 1, i=2,3} \frac{F_{n}}{2}\left|d_{n}^{(i)}\right|^{2} \geq 0 .
\end{gathered}
$$

Therefore, $d_{n}^{(i)}=0$ for all $n$ and $i$ at small $m_{\mathrm{TF}}$ and we obtain $\mathrm{U}(1)$ symmetric phase $(\Delta(p)=0)$. This phase was studied in the Hartree-Fock approximation. It was shown that the compressible striped state is favored in this phase 18, 19, 13. At large $m_{\mathrm{TF}}$ for $l=1$ and $2, \eta_{\mathbf{X}}=0$ for $\mathbf{X} \neq 0$ and we obtain a pairing phase. Thus, in the Hartree-FockBogoliubov approximation, the stripe phase makes transition to the pairing phase for $m_{\mathrm{TF}}>1.0 \sim 2.0$. In this pairing phase, $\xi=0$ and the energy spectrum of the quasiparticle becomes $|\Delta(p)|$. Hence, there are at least four gapless points in the Brillouin zone for the obtained paired state. This peculiar result conflicts with the experiment [⿶] and numerical calculations [5.6], in which the paired state has an energy gap. Furthermore we find that the self-consistency for $m_{\mathrm{TF}}$ (3.21) is not satisfied, which goes to infinity as iterating the numerical calculations. This means that the fluctuation is too large and the mean field solution is not stable. In the next section, we change the summation of infinite diagrams of the Fermion self-energy part and obtain a self-consistent paired state with an energy gap.

\section{EFFECTIVE HAMILTONIAN FOR THE STRIPED AND PAIRED STATE}

In this section, we present an effective Hamiltonian which has a striped state as the normal state and paired state as the U(1) symmetry breaking state. We focus our argument on the half-filled second Landau level space, that is $l=1, \nu=1+1 / 2$. We use a tricky technique to find a self-consistent solution for the paired state with an energy gap. The most hardest problem in the quantum Hall system is to derive a low-energy effective theory from the microscopic Hamiltonian, because we do not know a small parameter used in the perturbation expansion a priori. Therefore we have to choose a starting point by consideration of symmetry and physical intuition based on experiments. In the present case, we maintain the translational symmetry on the von Neumann lattice and choose the striped state as the normal state.

Let us divide the mean field Hamiltonian (2.26) into two parts as

$$
\begin{aligned}
H_{\text {mean }} & =H_{0}+H_{1}, \\
H_{1} & =\int_{\mathrm{BZ}} \frac{d^{2} p}{(2 \pi)^{2}} t \cos p_{y} a^{\dagger}(p) a(p),
\end{aligned}
$$

and $H_{1}$ is treated as perturbation to $H_{0}=-H_{1}+H_{\text {mean }}$. Self-consistency is imposed for $H_{0}$ first and correction from $H_{1}$ is included next. $H_{1}$ represents the anisotropy in the stripe state or normal state in which the uniform direction is chosen in the $y$ direction. In the striped state at the half-filling, the Fermi sea is formed at $\left|p_{y}\right|<\pi / 2$, which is shown in Ref. 13 In this case the Fermi surface (line) is formed at $p_{y}= \pm \pi / 2$. It is expected that the paired state possesses the same anisotropy as the normal state. In $H_{0}$, the nearest-neighbor $(\mathrm{N}-\mathrm{N})$ hopping parameter becomes $t-2 \xi_{(0,1)}=t+t_{(0,1)}$. Then the self-consistent hopping parameter satisfies

$$
t_{\mathbf{X}}=-2 \xi_{\mathbf{X}}\left[\left\{t \delta_{m, n}^{0,1}+t_{(m, n)}\right\}, \Delta(p)\right] .
$$

The N-N hopping parameter is renormalized as $t^{\prime}=t+t_{(0,1)}$ in $H_{0}$. Including the first order correction of $H_{1}$, the effective hopping parameter becomes $t_{\mathrm{eff}}=t^{\prime}-t=t_{(0,1)}$. The parameter $t$ controls the effective N-N hopping strength dynamically. The magnitude of $t_{\text {eff }}$ corresponds to the strength of the stripe order. 
We truncate the expansions of Eqs. (3.1) and (3.15) and calculate the self-consistent solution by iteration of numerical calculations until we obtain convergence. Considering only the lowest and next relevant terms, the effective Hamiltonian for the quasiparticle in the striped and paired state is given by

$$
H_{\mathrm{eff}}=\int_{\mathrm{BZ}} \frac{d^{2} p}{(2 \pi)^{2}}\left[\varepsilon_{\mathrm{eff}}(p) a^{\dagger}(p) a(p)+\frac{1}{2} \Delta_{\mathrm{eff}}(p) a^{\dagger}(-p) a^{\dagger}(p)+\frac{1}{2} \Delta_{\mathrm{eff}}^{*}(p) a(p) a(-p)\right],
$$

where the effective hopping potential and effective gap potential are given by

$$
\begin{aligned}
\varepsilon_{\mathrm{eff}}(p) & =-t_{\mathrm{eff}} \cos p_{y}-t_{(0,3)} \cos 3 p_{y}, \\
\Delta_{\mathrm{eff}}(p) & =c_{1}^{(3)} \psi_{1}^{(3)}(p)+c_{3}^{(3)} \psi_{3}^{(3)}(p) .
\end{aligned}
$$

In using Eqs. (4.5) and (4.6), $\psi_{n}^{(2)}$ and $\cos \left((2 m+1) p_{x}+n p_{y}\right)$ terms are irrelevant and $\cos \left(2 m p_{x}+2 n p_{y}\right)$ terms induced in $\varepsilon_{\text {eff }}$ give a negligible correction to the numerical results. The $t$ dependence of $t_{\text {eff }}$ is determined self-consistently by $t_{\mathrm{eff}}=-2 \xi_{(0,1)}\left[t+t_{\mathrm{eff}}, t_{(0,3)}, c_{1}^{(3)}, c_{3}^{(3)}\right]$. The $t$ dependence is implicit in the effective theory. The hopping parameters $t_{(0, n)}$ and gap potential $\Delta_{\text {eff }}(p)$ depend on $t_{\text {eff }}$ explicitly. We use the following approximation

$$
m_{\mathrm{TF}}=\frac{1}{\pi t_{\mathrm{eff}}},
$$

which is satisfied in the stripe phase with only the N-N hopping term. We checked that Eq. (4.7) was good approximation using the obtained convergent solutions.

Chemical potential $\mu$ is determined so that the filling factor $\nu_{1}$ is equal to $1 / 2$. Note that $\mu$ includes the on-site term in $H_{\text {mean }}$. We find that $\mu$ is negative small number on the order of $10^{-4}$ at most. The gap potential $\Delta_{\text {eff }}(p)$ depends on $t_{\text {eff }}$. Figure (7) shows the $t_{\text {eff }}$ dependence of the energy gap $\Delta E=\min (2 E(p))$ at $\nu=1+1 / 2$. The maximum

value of the energy gap is 0.027 at $t_{\text {eff }}=0.03$. In this case, $t_{(0,3)}=-0.0003, c_{1}^{(3)}=0.0104, c_{3}^{(3)}=-0.0018$, and $\mu=-0.0006$. The energy spectrum of the quasiparticle at $t_{\mathrm{eff}}=0.03$ is shown in Fig. (8). The absolute value of the gap potential at $t_{\text {eff }}=0.03$ is shown in Fig. (9). The excitation energy $E(p)$ becomes small around $p_{y}= \pm \pi / 2$, which is the Fermi surface of the striped state. The transition to the stripe phase is continuous and very smooth. Near the transition point, the behavior of energy gap is approximated by $t_{\mathrm{eff}} e^{-2 \pi t_{\mathrm{eff}} /\left|F_{1}\right|}$, whose non-perturbative dependence on the coupling is well-known in the BCS theory. At $m_{\mathrm{TF}}=m_{c} \approx 1.4, F_{1}$ behaves as $\alpha\left(m_{c}-m_{\mathrm{TF}}\right)$ as seen in Fig. (4), and the energy gap approaches to zero as

$$
\Delta E \propto t_{\mathrm{eff}} e^{-\frac{2 \pi t_{\mathrm{eff}}^{2}}{\alpha m_{c}\left(t_{c}-t_{\mathrm{eff}}\right)}}, \quad \text { for } t_{\mathrm{eff}}<t_{c}
$$

where $t_{c} \approx 1 / \pi m_{c} \approx 0.2$. The energy gap is extremely small at $0.1<t_{\mathrm{eff}}<t_{c}$. At $t_{\mathrm{eff}}>t_{c}$, the gap potential vanishes and the compressible striped state is realized. The spectrum of the striped state is uniform in the $p_{x}$ direction. This state is regarded as a collection of the one-dimensional lattice Fermion systems and leads to the anisotropy of the magnetoresistance 13.

Inspecting the energy spectrum of quasiparticle in the pairing phase, we find a crossover phenomenon at $t_{\mathrm{eff}} \sim 0.01$, that is, the minimum excitation energy $\min (E(p))$ is placed around $p_{y}= \pm \pi / 2$ at $0.01<t_{\mathrm{eff}}<t_{c}$, whereas at $0<t_{\text {eff }}<0.01$, placed around $p_{y}=0$ and $\pi$. We call the latter case the gap-dominant pairing phase. In this phase, the low-energy excitation occurs around zeros of the gap potential and the the energy gap is given by $2 \xi(0)$. Therefore the $t_{\text {eff }}$ dependence of energy gap $\Delta E$ becomes linear at $0<t_{\text {eff }}<0.01$ The energy spectrum at $t_{\text {eff }} \sim 0.01$ is shown in Fig. (10). As seen in this figure, the spectrum is close to the flatband and the stripe order is weakened compared with Fig. (8).

\section{SUMMARY AND DISCUSSION}

We propose an effective Hamiltonian which describes the striped and paired state at the half-filled Landau levels. The gap potential and screening mass are determined self-consistently in the Hartree-Fock-Bogoliubov approximation scheme. The energy spectrum of the quasiparticle and energy gap are calculated numerically.

The very smooth transition from pairing to stripe phase occurs at $t_{\mathrm{eff}}=t_{c}$. In other words, the stripe order strongly suppresses the pairing order at $0.1<t_{\mathrm{eff}}<t_{c}$. The energy gap becomes maximum at $t_{\mathrm{eff}}=0.03$ and the maximum value is $0.027 q^{2} / a=0.01 q^{2} / l_{B}\left(l_{B}=\sqrt{\hbar / e B}\right)$ which is the same order as Morf's value [5]. The experimental value 
at $\nu=5 / 2$ is smaller than the theoretical one by an order of magnitude $\llbracket$. This difference could be explained by effects of disorder and finite thickness of $2 \mathrm{D}$ layer, which reduce the energy gap [22]. The regions of small $t_{\mathrm{eff}}$ and $0.1<t_{\mathrm{eff}}<t_{c}$ have another possibility to explain the experiment. In our model, the crossover is obtained for small $t_{\text {eff }}$. This suggests that the stripe order is destroyed by the pairing order and the system becomes isotropic in the gap-dominant pairing phase.

The electric charge of the quasiparticle is a half of the electron charge and the Hall conductance is quantized as $e^{2} / 2 h$. The results are consistent with the recent experiment. However, we do not know whether correction to the quantized Hall conductance is finite or zero. Related to this subject, $U(1)$ breaking effect in the $P T$ violating system is studied in 21]. Whether the Hall conductance in the paired state is topological invariant or not is an interesting future problem.

It is possible to consider a pairing mechanism based on the composite fermion picture [23]. However, it is unclear that the composite fermion picture is applicable to the second and higher Landau levels. It rather seems that the compressible stripe state [18] which has an anisotropic Fermi surface is the normal state at the half-filled second and higher Landau levels in the Hartree-Fock approximation and numerical calculations of small systems [19, 13]. In fact, the transition from the paired state to striped state is observed in the presence of an inplane magnetic field [15]. Furthermore Ref. 24] suggests that the Chern-Simons gauge fluctuations are strongly pair breaking. Controversially, a superfluid state at the half-filling Hall state is proposed [25] on the basis of the dipolar liquid picture of the composite fermion [26].

Relation between our model and the Pfaffian state is not understood yet. Our effective Hamiltonian for the striped and paired state resembles the effective Hamiltonian proposed by Read and Green [27], which has the Pfaffian state in the weak pairing phase. However their normal state has the isotropic Fermi surface, which is reminiscent of the composite Fermion theory, and is different from our anisotropic one. The p-wave behavior seen in Eqs. (3.9) and (3.10), on the other hand, is in common with the Pfaffian state. In our case, $\Delta(p)$ becomes $c_{x} p_{x}+i c_{y} p_{y}, c_{y} \gg c_{x}$ in the long wave length limit $p \rightarrow 0$.

More experimental and theoretical studies are needed to decide the symmetry of the gap potential and to understand the underlying physics at the half-filled second Landau level. To compare the theory with experiments quantitatively, effects of the Landau level mixing, finite temperature, finite thickness of 2D layer, and inplane magnetic field need to be included in the calculation. Furthermore, Josephson effect, vortex excitations, and edge states in our model are important future subjects.

\section{ACKNOWLEDGMENTS}

I would like to thank K. Ishikawa, T. Aoyama and especially J. Goryo for useful discussions. Part of this work was done when I took part in Tenth International Conference on Recent Progress in Many-Body Theories, Seattle. This work was partially supported by the special Grant-in-Aid for Promotion of Education and Science in Hokkaido University provided by the Ministry of Education, Science, Sport, and Culture, the Grant-in-Aid for Scientific Research on Priority area (Physics of CP violation) (Grant No. 12014201), and the Grant-in aid for International Science Research (Joint Research 10044043) from the Ministry of Education, Science, Sports, and Culture, Japan.

[1] D. C. Tsui, H. L. Stormer, and A. C. Gossard, Phys. Rev. Lett. 48 (1982) 1559.

[2] R. B. Laughlin, Phys. Rev. Lett. 50 (1983) 1395.

[3] J. K. Jain, Phys. Rev. Lett. 63 (1989) 199; B. I. Halperin, P. A. Lee, and N. Read, Phys. Rev. B 47 (1993) 7312 ; Composite Fermion: A Unified View of the Quantum Hall Regime, edited by O. Heinonen (World Scientific, Singapore, 1998).

[4] R. L. Willett, J. P. Eisenstein, H. L. Stormer, D. C. Tsui, A. C. Gossard, and J. H. English, Phys. Rev. Lett. 59 (1987) 1776; W. Pan, J.-S. Xia, V. Shvarts, E. D. Adams, H. L. Stormer, D. C. Tsui, L. N. Pfeiffer, K. W. Baldwin, and K. W. West, ibid. 83 (1999) 3530.

[5] R. Morf, Phys. Rev. Lett. 80 (1998) 1505.

[6] E. H. Rezayi and F. D. M. Haldane, Phys. Rev. Lett. 84 (2000) 4685.

[7] G. Moore and N. Read, Nucl. Phys. B 360 (1991) 362; N. Read and G. Moore, Prog. Theor. Phys. Suppl. 107 (1992) 157.

[8] M. Greiter and F. Wilczek, Nucl. Phys. B 370 (1992) 577; M. Greiter, X.-G. Wen, and F. Wilczek, ibid. 374 (1992) 567; Phys. Rev. Lett. 66 (1991) 3205; M. Greiter, Prog. Theor. Phys. Suppl. 107 (1992) 145. 
[9] E. Fradkin, C. Nayak, A. Tsvelik, and F. Wilczek, Nucl. Phys. B 516 (1998) 704; E. Fradkin, C. Nayak, and K. Schoutens, ibid., 546 (1999) 711.

[10] F. D. M. Haldane and E. H. Rezayi, Phys. Rev. Lett. 60 (1988) 956, 1886(E).

[11] K. Ishikawa, N. Maeda, and T. Ochiai, Phys. Rev. Lett. 82 (1999) 4292.

[12] K. Ishikawa, N. Maeda, T. Ochiai, and H. Suzuki, Physica E 4 (1999) 37; K. Ishikawa and N. Maeda, Prog. Theor. Phys. 97 (1997) 507. N. Imai, K. Ishikawa, T. Matsuyama, and I. Tanaka, Phys. Rev. B 42 (1990) 10610.

[13] N. Maeda, Phys. Rev. B 61 (2000) 4766.

[14] M. P. Lilly, K. B. Cooper, J. P. Eisenstein, L. N. Pfeiffer, and K. W. West, Phys. Rev. Lett. 82 (1999) 394; R. R. Du, D. C. Tsui, H. L. Stormer, L. N. Pfeiffer, K. W. Baldwin, and K. W. West, Solid State Commun. 109 (1999) 389.

[15] W. Pan, R. R. Du, H. L. Stormer, D. C. Tsui, L. N. Pfeiffer, K. W. Baldwin, and K. W. West, Phys. Rev. Lett. 83 (1999) 820; M. P. Lilly, K. B. Cooper, J. P. Eisenstein, L. N. Pfeiffer, and K. W. West, ibid. 83 (1999) 824.

[16] A. M. Perelomov, Teor. Mat. Fiz. 6 (1971) 213 [Theor. Math. Phys. 6 (1971) 156]; V. Bargmann, P. Butera, L. Girardello, and J. R. Klauder, Rep. Math. Phys. 2 (1971) 221.

[17] Strictly speaking, the Wannier basis is weekly localized state. That is, it is normalizable, but its standard deviation of position is infinite. See Ref. [12].

[18] A. A. Koulakov, M. M. Fogler, and B. I. Shklovskii, Phys. Rev. Lett. 76 (1996) 499; M. M. Fogler, A. A. Koulakov, and B. I. Shklovskii, Phys. Rev. B 54 (1996) 1853; R. Moessner and J. T. Chalker, ibid. 54 (1996) 5006.

[19] T. Jungwirth, A. H. MacDonald, L. Smarcka, and S. M. Girvin, Phys. Rev. B 60, 15574 (1999); E. H. Rezayi, F. D. M. Haldane, and K. Yang, Phys. Rev. Lett. 83 (1999) 1292; T. Stanescu, I. Martin, and P. Phillips, Phys. Rev. Lett. 84 (2000) 1288 .

[20] F. D. M. Haldane, Phys. Rev. Lett. 51 (1983) 605.

[21] J. Goryo and K. Ishikawa, Phys. Lett. A 260 (1999) 294.

[22] R. L. Willett, H. L. Stormer, D. C. Tsui, A. C. Gossard, and J. H. English, Phys. Rev. B 37 (1988) 8476.

[23] T. Morinari, Phys. Rev. Lett. 81 (1998) 3741.

[24] N. E. Bonesteel, Phys. Rev. Lett. 82 (1999) 984.

[25] G. Baskaran, Physica B 212 (1995) 320.

[26] N. Read, Semicond. Sci. Technol. 9 (1994) 1859; V. Pasquier and F. D. M. Haldane, Nucl. Phys. B 516 (1998) 719.

[27] N. Read and D. Green, Phys. Rev. B 61 (2000) 10267.

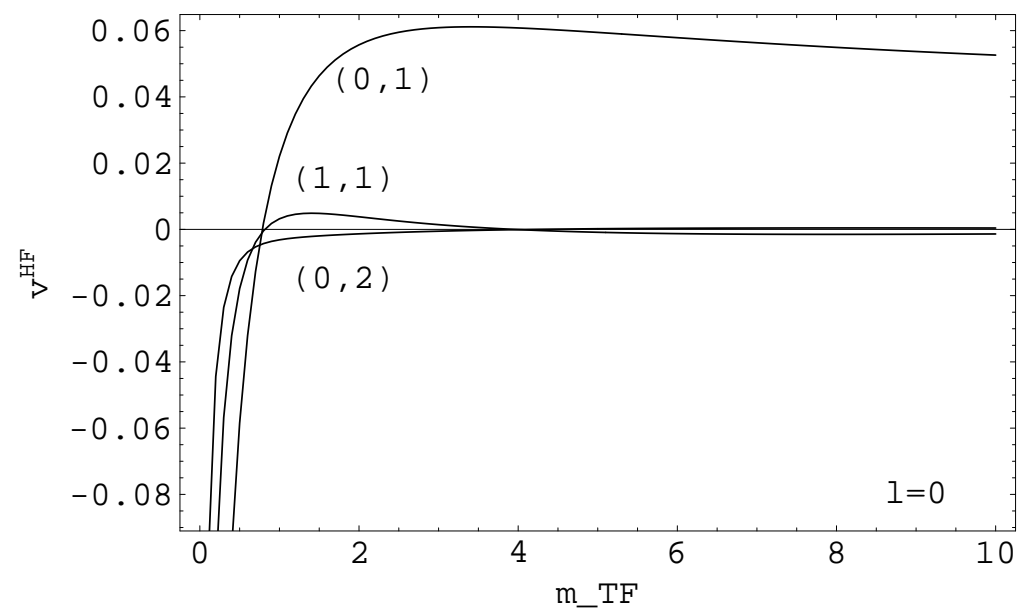

Fig. 1. The $m_{\mathrm{TF}}$ dependence of $v^{\mathrm{HF}}(0,1), v^{\mathrm{HF}}(1,1)$, and $v^{\mathrm{HF}}(0,2)$ for $l=0$. The unit of energy is $q^{2} / a$. The same unit is used in all other figures. 


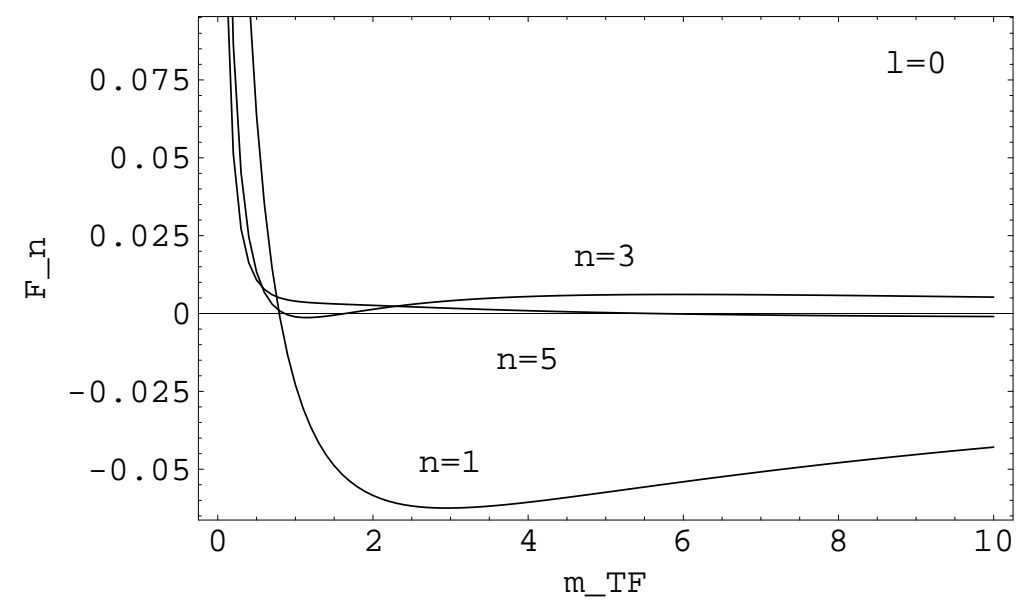

Fig. 2. The $m_{\mathrm{TF}}$ dependence of pseudopotentials $F_{1}, F_{3}$, and $F_{5}$ for $l=0$.

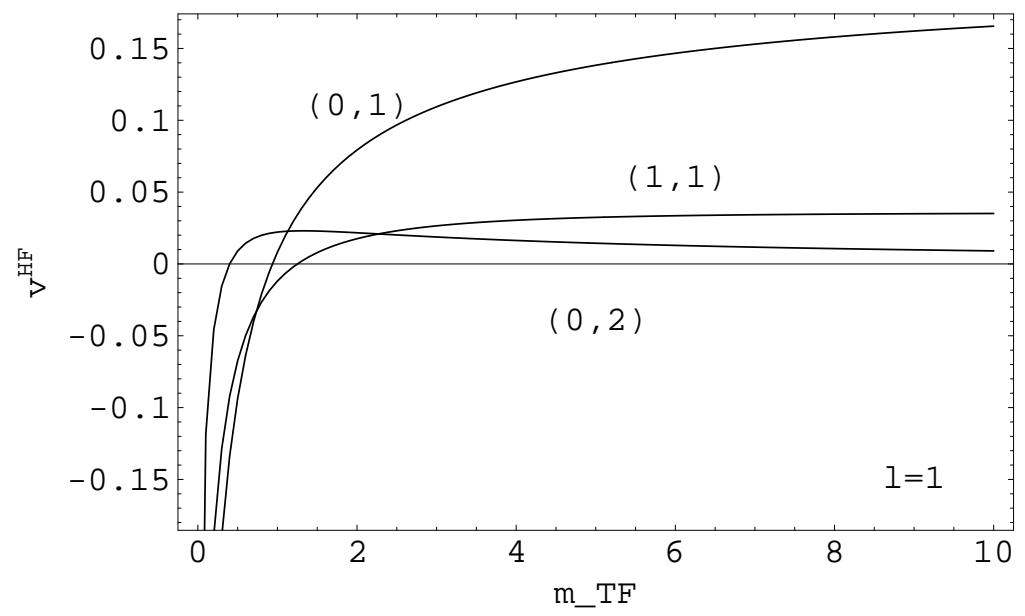

Fig. 3. The $m_{\mathrm{TF}}$ dependence of $v^{\mathrm{HF}}(0,1), v^{\mathrm{HF}}(1,1)$, and $v^{\mathrm{HF}}(0,2)$ for $l=1$.

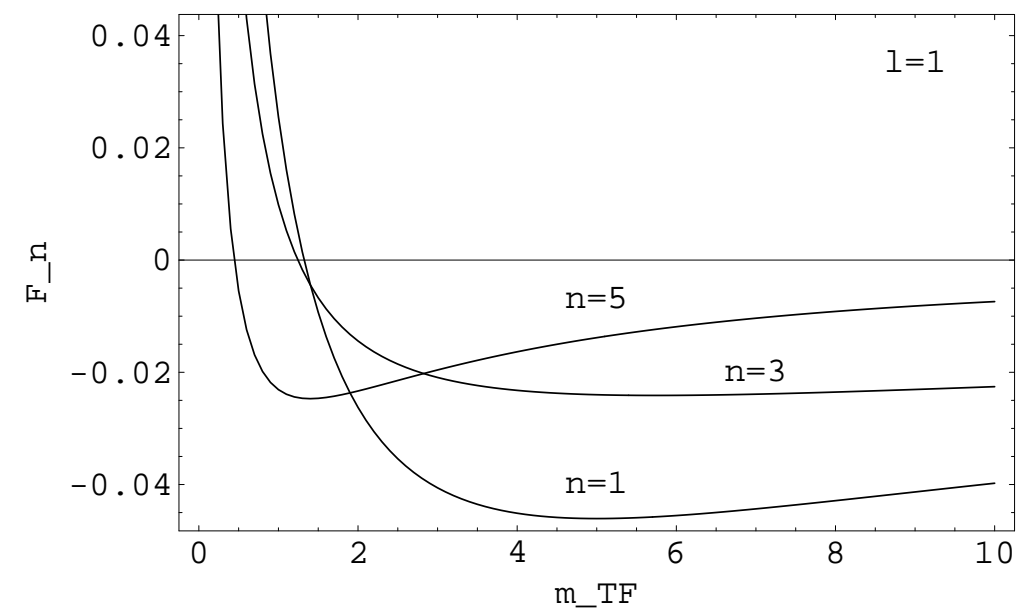

Fig. 4. The $m_{\mathrm{TF}}$ dependence of pseudopotentials $F_{1}, F_{3}$, and $F_{5}$ for $l=1$. 


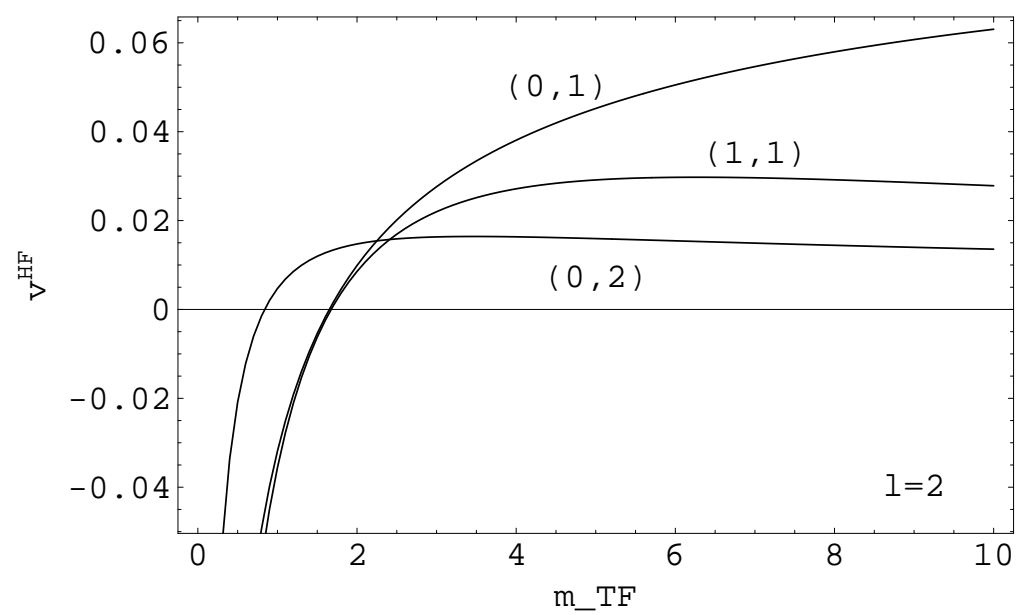

Fig. 5. The $m_{\mathrm{TF}}$ dependence of $v^{\mathrm{HF}}(0,1), v^{\mathrm{HF}}(1,1)$, and $v^{\mathrm{HF}}(0,2)$ for $l=2$.

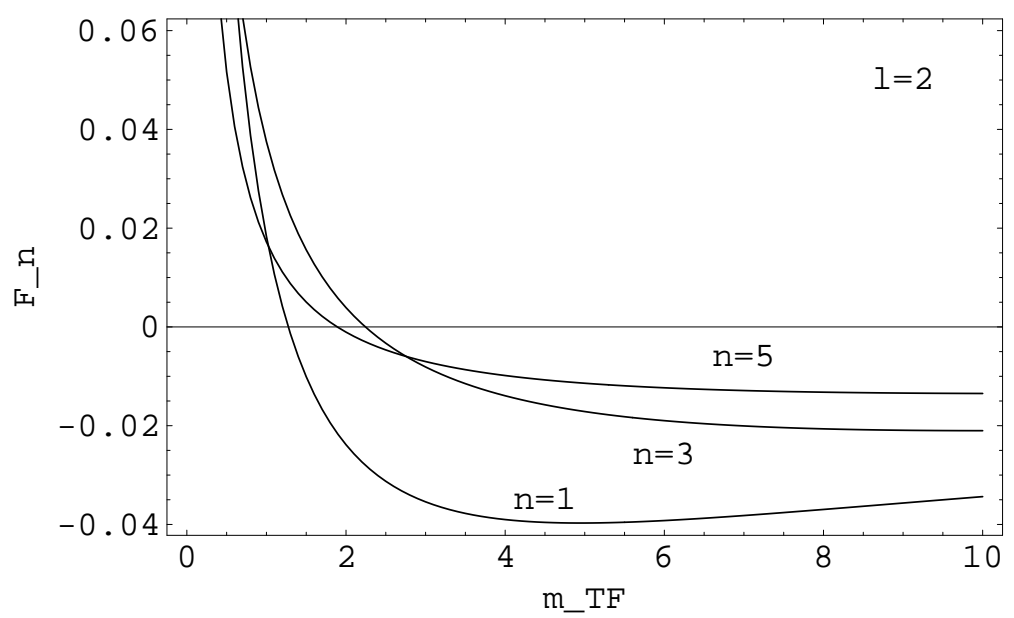

Fig. 6. The $m_{\mathrm{TF}}$ dependence of pseudopotentials $F_{1}, F_{3}$, and $F_{5}$ for $l=2$.

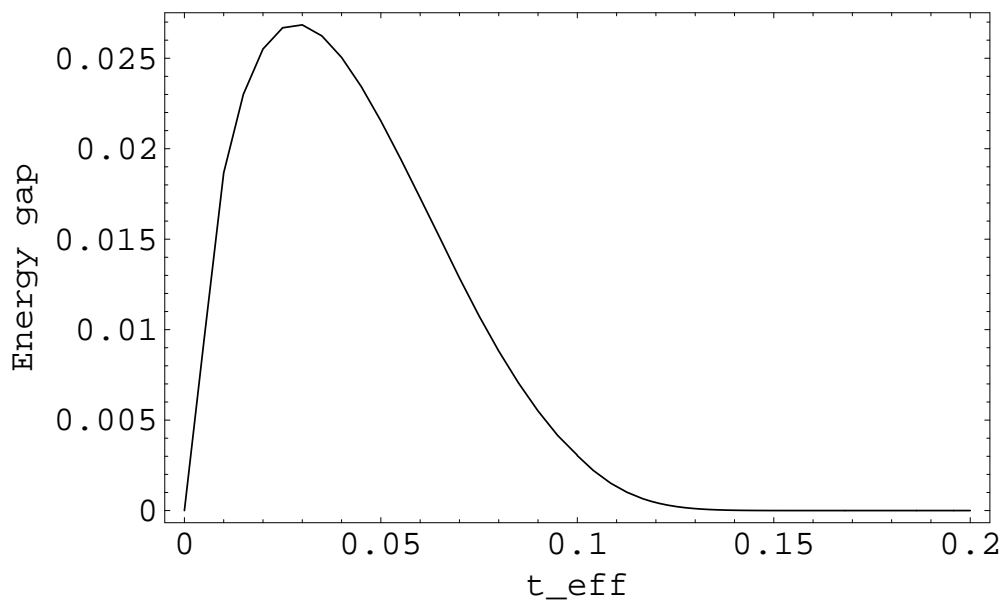

Fig. 7. The $t_{\text {eff }}$ dependence of the energy gap $\Delta E$ for $l=1$. Crossover occurs at $t_{\text {eff }} \approx 0.01$. The energy gap becomes maximum at $t_{\mathrm{eff}} \approx 0.03$ and vanishes at $t_{\mathrm{eff}} \approx 0.2$. 


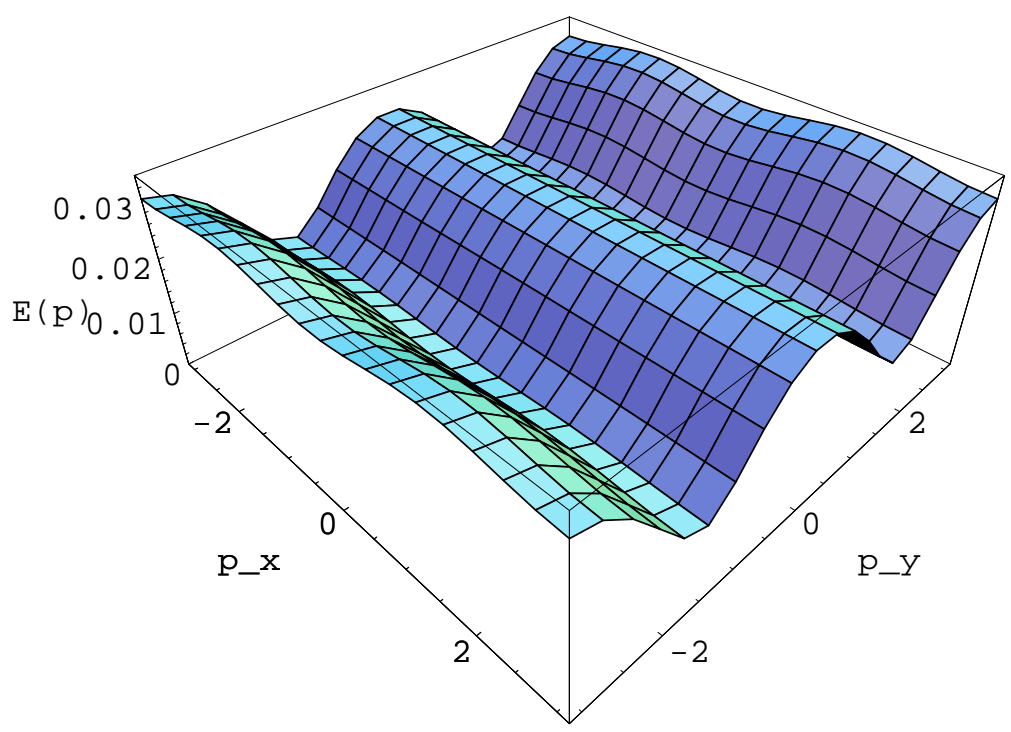

Fig. 8. Spectrum of the quasiparticle $E(p)$ at $t_{\mathrm{eff}}=0.03$ for $l=1$.

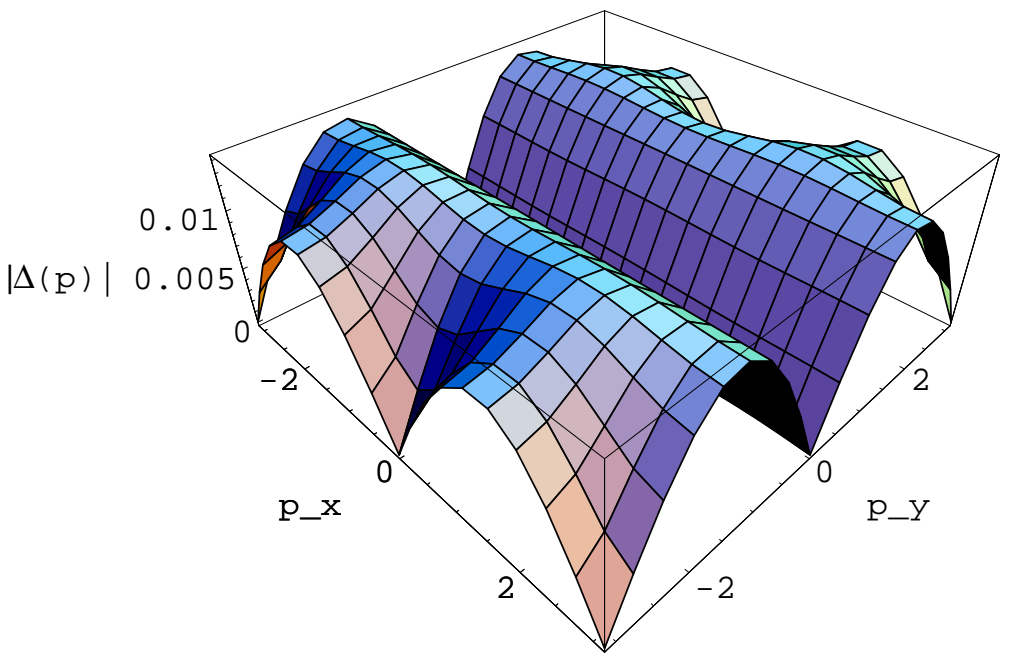

Fig. 9. Absolute value of the gap potential $|\Delta(p)|$ at $t_{\mathrm{eff}}=0.03$ for $l=1$. 


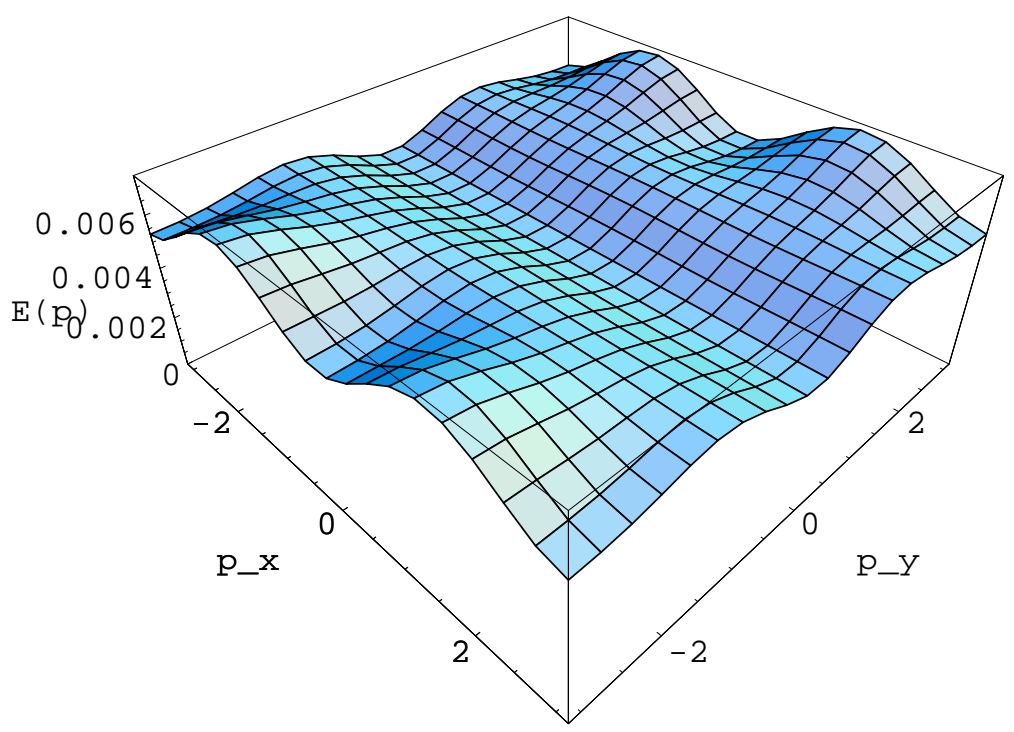

Fig. 10. Spectrum of the quasiparticle $E(p)$ at $t_{\mathrm{eff}}=0.005$ for $l=1$. 